\title{
Variabilidade espacial da altitude elipsoidal determinada por GNSS, ASTER GDEM e SRTM
}

\author{
Spatial variability of ellipsoidal altitude determined by GNSS, ASTER GDEM and SRTM
}

\author{
Rafael Noetzold ${ }^{1}$ \\ Marcelo de Carvalho Alves ${ }^{2}$ \\ Rogério Antonio Gallon ${ }^{1}$ \\ Maicon José Peres Arruda Oliveira ${ }^{1}$
}

\begin{abstract}
${ }^{1}$ Departamento de Solos e Engenharia Rural, Universidade Federal de Mato Grosso - UFMT, Faculdade de Agronomia, Medicina Veterinária e Zootecnia. Av. Fernando Correa da Costa, n 2367, Boa Esperança. Cep 780860 900. Cuiabá, MT, Brasil. ${ }^{2}$ Departamento de Engenharia, Universidade Federal de Lavras - UFLA. Campus Universitário. Cep 37200-000. Lavras, MG, Brasil

rafael_noetzold@hotmail.com, marcelo.alves@deg.ufla.br, ragallon@yahoo.com.br, yaomicon@hotmail.com
\end{abstract}

\begin{abstract}
RESUMO - Foi estudada a variabilidade espacial da altitude obtida por GNSS, ASTER GDEM e SRTM. Foram coletados com receptores Global Navigation Satellite Systems (GNSS) 3884 dados de altitude, e utilizados para comparação, os dados de altitude dos modelos SRTM e ASTER, extraídos pela técnica do vizinho mais próximo. A análise geoestatística foi feita por meio de ajustes de semivariograma esférico, exponencial e gaussiano, sendo utilizado o critério de informação de Akaike, a soma de quadrados e o desvio padrão da krigagem para escolha do melhor modelo de ajuste. Em seguida, efetuou-se a interpolação dos dados por krigagem ordinária. A geoestatística possibilitou caracterizar a estrutura e a magnitude de dependência espacial da altitude. Ocorreu dependência espacial para os três conjuntos de dados estudados. Para os dados do GNSS, ASTER e SRTM, o melhor modelo foi o esférico. Os maiores erros da krigagem foram obtidos pelos dados SRTM e, os menores erros pelos dados GNSS.
\end{abstract}

Palavras-chave: Geoestatística, Critério de informação de Akaike, Modelo esférico, Krigagem.

ABSTRACT - It was studied the spatial variability of elevation obtained by GNSS, SRTM and ASTER GDEM. And 3,884 altitude data were collected with receptors Global Navigation Satellite Systems (GNSS). Altitude data from SRTM and ASTER models extracted by the nearest neighbor technique were used for comparison. The geostatistical analysis was performed by adjusting semivariogram spherical, exponential and gaussian being used the Akaike information criterion, the sum of squares value and kriging standard deviation to select the best fit model. Then, the interpolation of the data by ordinary kriging. Geostatistical possible to characterize the structure and magnitude of spatial dependence of altitude. There was spatial dependence for the three data sets studied. For GNSS data, ASTER and SRTM the best model is the spherical. The largest error of kriging were obtained by SRTM data and the least error for GNSS data.

Keywords: Geostatistics, Akaike information criterion, Spherical model, Kriging.

\section{INTRODUÇÃO}

A representação digital da superfície do terreno é fator significativo na busca de ferramentas auxiliares à organização espacial do ambiente (BARBOSA et al., 2012).

A utilização de informações obtidas por meio do sensoriamento remoto ocorre nas mais diversas áreas da ciência como a do meio ambiente, da geomorfologia, da hidrologia, de estudos urbanos etc. Os produtos gerados pelo sensoriamento remoto são expressos de diversas formas, entre elas destaca-se o Modelo Digital de Elevação (MDE).

Os MDEs podem ser gerados com base nos dados provenientes de produto de sensoriamento remoto. Após várias etapas de seleção, correção e processamento desses dados, torna-se possível a geração de um MDE. Entre os MDEs, atualmente o Advanced Spaceborne Thermal
Emission and Reflection Radiometer (ASTER), sendo que os dados GDEM são obtidos a partir do par estereoscópico de imagens do infravermelho próximo (CAMARGO et al., 2009), e o Shuttle Radar Topography Mission (SRTM) são os mais utilizados.

O conhecimento da altitude em áreas urbanas contribui de forma expressiva para o planejamento do uso e ocupação do solo, pois é uma das informações essenciais para a elaboração de projetos de construção civil e planejamento territorial. A obtenção desses dados de altitude pode ser feita por levantamento local, com a utilização de equipamentos topográficos, ou de forma indireta, utilizando os dados remotos.

Para obtenção de informações da topografia de forma indireta, os dados obtidos do SRTM e ASTER GDEM (Global Digital Elevation Model), e de receptor Global Navigation Satellite Systems (GNSS), são empregados em estudos de altitude. 
Após a disponibilidade dos dados SRTM, vários estudos os utilizaram em aplicações topográficas $(\mathrm{KOCH}$; LOHMANN, 2000; SMITH; SANDWELL, 2003; FALORNI et al., 2005; CHAGAS et al., 2010). É importante destacar que, dependendo da finalidade do uso da altitude, sua acurácia deve ser levada em consideração. Koch e Lohmann (2000) citaram que as possíveis fontes de erros dos dados SRTM podem ser divididas na caracterização dos parâmetros In $S A R$ durante a aquisição de dados, no processamento e nas influências da vegetação e da cobertura do solo.

Estudos conduzidos por Stevens et al. (2004) apontaram melhor representação da topografia de vulcões ativos com dados do ASTER, sendo que os dados do SRTM foram considerados imprecisos, pois a topografia pode mudar significativamente após a atividade vulcânica. Mas Arefi e Reinartz (2011) afirmaram que os dados obtidos por ASTER GDEM incluem artefatos e outliers que diminuem a qualidade e, para resolver esse problema, desenvolveram um algoritmo para melhorar a informação. Os autores observaram significativa melhora, pois a maior parte dos erros e artefatos foram removidos.

Por outro lado, Kervyn et al. (2008) citaram vantagens e limitações do SRTM e ASTER em estudo sobre mapeamento topográfico vulcânico. Para a avaliação da precisão topográfica global são necessários estudos desses produtos envolvendo dados de verdade terrestre. Para tanto o GNSS pode fornecer resulados mais precisos e acurados.

As técnicas da geoestatística permitem avaliar os dados altimétricos oriundos de diversas fontes. Umas dessas técnicas, a krigagem, pode ser utilizada com sucesso na elaboração de MDE (HENGL et al., 2008). Ferramentas geoestatísticas podem contribuir para o melhor entendimento da modelagem da altitude. Neste sentidio, objetivou-se avaliar a variabilidade espacial da altitude obtida por dados ASTER GDEM, GNSS e SRTM.

\section{MATERIAL E MÉTODOS}

A pesquisa foi realizada no campus de Cuiabá da Universidade Federal de Mato Grosso (UFMT), durante o período de maio a agosto de 2011.

Foram definidos 27 pontos delimitando a área do campus, sendo utilizado o posicionamento relativo estático para gerar esses pontos e posteriormente o posicionamento cinemático com pós processamento dos dados (GHILANI; WOLF, 2012).

Primeiramente foi feito um rastreamento estático seguido de pós processamento dos dados com a RBMC de Cuiabá. Nesse caso, cada receptor permaneceu oito horas para gerar os pontos com precisão milimétrica de coordenadas geodésicas, sendo que os dados foram gravados a cada 15 segundos. $\mathrm{O}$ pós processamento foi $\mathrm{o}$ posicionamento por ponto preciso (PPP) com órbita precisa, após duas semanas da realização do levantamento.

Foi utilizado receptores GPS+ Hiper da Topcon Positioning System de dupla frequência, os quais podem receber e processar os sinais L1 e L2 e, permitem acesso aos satélites GPS e GLONASS. Esses receptores foram configurados com o datum WGS 84, projeção UTM e zona $21 \mathrm{~S}$.

Em seguida, foi feito um levantamento cinemático Real Time Kinematic (RTK), com precisão horizontal e vertical de $10 \mathrm{~mm}$ e $20 \mathrm{~mm}+1,5 \mathrm{ppm}$ x D respectivamente, sendo a base instaladas nos pontos que foram corrigidos via PPP, denominado PRTK (RTK pós processado) (GHILANI; WOLF, 2012), essa correção foi feita por meio da utilização do programa Topcon Tools. A base foi instalada em sete pontos estratégicos, sendo registrados dados a cada 5 segundos, desde que houvesse o sinal fixo entre a base e o rover, ao longo de vias, meio fio, pista de atletismo, quadras de esportes, campo de futebol, degraus de escadas, calçadas, pé direito de construções prediais, estacionamentos, corredores, com intuito de capturar os detalhes da área estudada e assim obter uma maior precisão dos dados. Foi coletado um total de 3884 pontos (Fig. 1).

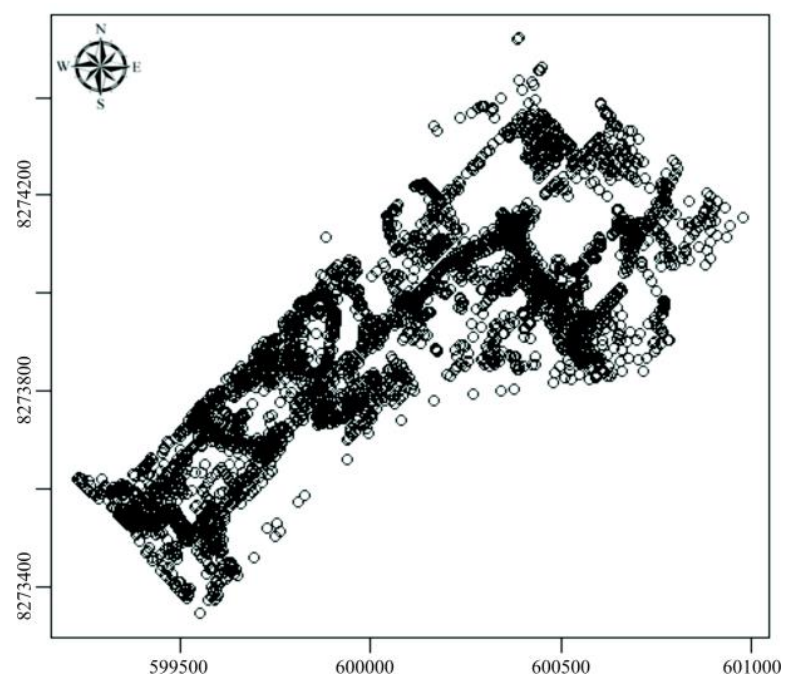

Figura 1 - Grid com indicação dos pontos amostrais.

A partir desses 3884 pontos, foi obtida a distância mínima e máxima entre os mesmos de $0,0014 \mathrm{~m}$ e $1827,18 \mathrm{~m}$, respectivamente e, posteriormente foi gerado o mapa de altimetria por meio da krigagem.

A confiabilidade média interna do processamento da altitude dos pontos de contorno e do levantamento cinemático RTK foi de $0,2047 \mathrm{~m}$ e $0,0449 \mathrm{~m}$, respectivamente.

Para gerar os dados altimétricos com os dados das imagens ASTER GDEM e SRTM foram coletados os valores da altitude a partir da latitude e longitude ao longo dos pontos coletados no campus, por meio de uma amostragem dessas imagens por uma malha de pontos de 30 x $30 \mathrm{~m}$ distribuída em ambos os produtos. $\mathrm{O}$ critério de amostragem foi determinado pelo algoritmo do vizinho mais próximo, o qual seleciona o valor do ponto mais próximo e não considera os valores dos pontos vizinhos. Esse método foi escolhido para não interpolar os dados e não alterar os valores obtidos em uma malha mais refinada do que as imagens ASTER e SRTM original. No 
caso de o ponto cair exatamente na interseção de pixels, a interpolação será constante.

Posteriormente foi feita a análise exploratória não espacial das três fontes de dados altimétricos, sendo calculados o valor mínimo, $1^{\circ}$ quartil, mediana, média, $3^{\circ}$ quartil, valor máximo, desvio padrão, curtose, assimetria e coeficiente de variação. Também foi feita a relação entre cada modelo de obtenção da altitude, representado por gráficos, utilizando o programa SigmaPlot. Foram calculadas também a correlação de Pearson $(\mathrm{P}=0,05)$ e a raiz quadrada do erro médio quadrático (RMSE), obtida pela Eq. 1.

$$
R M S E=\sqrt{\frac{1}{n} \sum(d)^{2}}
$$

sendo que,

- $d$ - corresponde à diferença de elevação entre dois MDEs avaliados;

- $n$ - corresponde ao valor de pares de dados.

A dependência espacial da altitude foi feita por meio de ajustes de semivariogramas, com base na pressuposição de estacionariedade da hipótese intrínseca. O semivariograma experimental foi estimado de acordo com o estimador clássico (BURROUGH; MCDONNELL, 1998), conforme a Eq. 2.

$$
y(h)=\frac{1}{2 N(h)} \sum_{i=1}^{N(h)}\left[Z\left(x_{i}\right)-Z\left(x_{i}+h\right)\right]^{2}
$$

sendo que,

- $y(h)$ - semivariância estimada a partir dos dados experimentais;

- $N(h)$ - números de pares experimentais das observações $Z\left(x_{i}\right), \quad Z\left(x_{i}+h\right)$ separados pela distância $h$.

O método de ajuste utilizado foi o método dos mínimos quadrados ordinários (OLS) (DIGGLE; RIBEIRO JUNIOR, 2007). Os modelos teóricos de semivariogramas isotrópicos utilizados foram definidos de acordo com Diggle e Ribeiro Junior (2007), por meio dos modelos: esférico, exponencial e gaussiano, representado pelas respectivas Eqs. 3, 4 e 5.

$$
\begin{aligned}
& \rho(u)=\left\{1-\frac{3}{2}(u / \phi)+\frac{1}{2}(u / \phi)^{3} \begin{array}{l}
: 0 \leq u \leq \phi \\
0: u>\phi
\end{array}\right. \\
& \rho(u)=\exp \left\{-(u / \phi)^{k}\right\} \\
& \gamma(\mathrm{h})=\exp \left\{-\left(\phi^{2} / u^{2}\right)\right\}
\end{aligned}
$$

sendo que,

$$
\begin{aligned}
& \text { - } \phi \text { - é o alcance; } \\
& \text { - } u \text { - é a distância. }
\end{aligned}
$$

A escolha do melhor modelo foi feita utilizando o critério de informação de Akaike (Akaike's Information Criterion - AIC) (MELLO et al., 2005) por meio da Eq. 6; o menor valor da soma de quadrados e o menor erro da krigagem.

$$
A I C=-2 l+2 p
$$

sendo que,

- $l$ - é o ln da função de verossimilhança (loglikelihood);

- $p$ - é o número de parâmetros do modelo considerado.

Conforme Mello et al. (2005), o menor AIC representa o melhor modelo. Posteriormente, foram feitas a interpolação por krigagem ordinária gerando uma imagem com gride de $30 \mathrm{~m}$ para cada fonte de dados altimétricos, e a confecção dos mapas referente ao desvio padrão da krigagem. Todas as análises geoestatísticas foram realizadas no software $\mathrm{R}$ com o pacote geoR (DIGGLE; RIBEIRO JUNIOR, 2007).

\section{RESULTADOS E DISCUSSÃO}

Por meio da estatística descritiva, observou-se que os valores de média e mediana foram próximos. A curtose apresentou distribuição com curva platicúrtica para ambos os conjuntos de dados da altitude. Os valores de assimetria estão próximos de zero, tendo os dados obtidos por GNSS e SRTM distribuição levemente assimétrica à direita, enquanto, os dados do ASTER têm uma leve assimetria à esquerda. Observou-se também menor diferença entre o valor máximo e o mínimo da altitude no modelo SRTM e maior diferença nos dados obtidos pelo GNSS (Tab. 1).

Tabela 1- Estatística descrita referente aos valores de altitude do campus da UFMT, obtidos por meio de GNSS e dos modelos ASTER GDEM e SRTM.

\begin{tabular}{lrrr}
\hline & \multicolumn{3}{c}{ Modelos } \\
\cline { 2 - 4 } Índices estatísticos & GNSS & ASTER & SRTM \\
\hline Mínimo & 155,34 & 160,00 & 164,00 \\
$1^{\circ}$ quartil & 167,30 & 168,00 & 171,00 \\
Mediana & 172,29 & 174,00 & 176,00 \\
Média & 172,32 & 173,63 & 176,20 \\
$3^{\circ}$ quartil & 176,50 & 179,00 & 181,00 \\
Máximo & 187,68 & 186,00 & 189,00 \\
Curtose & $-0,81$ & $-1,05$ & $-0,99$ \\
Assimetria & 0,20 & $-0,13$ & 0,07 \\
Desvio padrão & 6,58 & 6,18 & 7,01 \\
CV (\%) & 3,82 & 3,56 & 3,98 \\
\hline
\end{tabular}

$\overline{\mathrm{CV}}$ - coeficiente de variação.

Os valores de coeficiente de variação foram menores que $4 \%$, resultado, provavelmente, devido à quantidade de pontos amostrais de cada conjunto de dados testado. Com base na observação do valor máximo e mínimo de cada conjunto de dados, foi possível verificar variação 
entre 25 a 32,34 m (Tab. 1). Conforme a classificação proposta por Warrick e Nielsen (1980) para o coeficiente de variação, a altitude observada para os três conjuntos de dados apresentou baixa variabilidade.

Com base na análise não espacial feita com os dados de altitude, assumiu-se que as distribuições podem ser consideradas suficientemente simétricas e com caudas não alongadas (Tab. 1), favorecendo assim a construção de semivariogramas.

Os valores referentes à raiz quadrada do erro médio quadrático para GNSS e ASTER $(2,94)$, GNSS e SRTM $(3,86)$, ASTER e SRTM $(4,66)$ foram aumentando, estando a melhor similaridade entre o conjunto de dados GNSS e ASTER. Provavelmente, devido ao tamanho do pixel proveniente dessas plataformas e também à declividade praticamente sem mudança abrupta, os dados oriundos do ASTER e SRTM representaram com qualidade inferior a altitude quando comparados aos dados do GNSS. Na Fig. 2 estão representadas as regressões lineares dos dados de altitutde entre GNSS, ASTER e SRTM, cujo os valores do coeficiente de determinação e da acurácia ficaram acima de 0,83. Para Felicísimo (1994), a qualidade de um MDE é dependente do tipo e da magnitude dos erros a ele relacionados. É importante considerar que os erros são inevitáveis, pois os MDEs são representações simplificadas da realidade, submetidas a um processo de generalização.

Observou-se correlação de Pearson entre os dados ASTER e SRTM de 0,91, entre GNSS e ASTER ( $\mathrm{r}=$ $0,92)$ e entre GNSS e SRTM $(r=0,93)$. Nikolakopoulos et al. (2006) compararam a elevação em duas regiões da Grécia por meio de dados do ASTER e do SRTM e observaram correlação de Pearson de 0,99 para ambas as regiões.

Foi possível verificar a existência de dependência espacial da altitude do campus da UFMT em todas as combinações testadas entre modelos e método (Tab. 2).

Tabela 2 - Parâmetros e coeficientes de semivariogramas ajustados aos dados de altitude do campus da UFMT obtido por GNSS, ASTER e SRTM.

\begin{tabular}{clrrrrrr}
\hline Modelo & $\begin{array}{r}\text { Efeito Pepita } \\
\left(\mathrm{c}_{0}\right)\end{array}$ & $\begin{array}{r}\text { Patamar } \\
\left(\mathrm{c}_{0}+\mathrm{c}\right)\end{array}$ & $\begin{array}{c}\text { Alcance } \\
(\mathrm{m})\end{array}$ & $\begin{array}{r}\text { Alcance } \\
\text { Prático }(\mathrm{m})\end{array}$ & AIC & $\begin{array}{c}\text { Soma de } \\
\text { quadrados }\end{array}$ \\
\hline GNSS & esférico & 0,00 & 6,66 & 317,60 & 317,60 & 5628,35 & 1,03 \\
GNSS & gaussiano & 0,60 & 6,63 & 145,57 & 251,96 & 8115,10 & 0,85 \\
GNSS & exponencial & 0,00 & 7,56 & 171,18 & 512,80 & 5611,82 & 1,22 \\
ASTER & esférico & 0,00 & 59,01 & 979,69 & 979,69 & 6042,93 & 244,86 \\
ASTER & gaussiano & 2,10 & 59,68 & 463,62 & 802,44 & 12954,62 & 167,41 \\
ASTER & exponencial & 0,00 & 69,65 & 578,83 & 1734,01 & 6917,14 & 514,66 \\
SRTM & esférico & 0,00 & 72,11 & 998,74 & 998,74 & 7992,44 & 260,21 \\
SRTM & gaussiano & 5,09 & 73,50 & 484,53 & 838,63 & 16622,92 & 181,27 \\
SRTM & exponencial & 0,00 & 106,28 & 832,15 & 2492,90 & 8297,49 & 461,05 \\
\hline
\end{tabular}

Para o conjunto de dados GNSS, foi necessário remover tendência de primeira ordem, para assim obter os melhores parâmetros dos semivariogramas, tendo o grau de dependência espacial, conforme Cambardella et al. (1994), sido classificado como forte para todas as combinações testadas (Tab. 2). Barbosa et al. (2012) também observaram forte dependência espacial para dados de elevação.

Pelo critério do AIC, pela soma de quadrados e, pelo menor erro da krigagem foi escolhido o modelo esférico para os três bancos de dados (Tab. 2), os quais foram usados para a construção de semivariogramas (Figs. 3A, 3B e 3C) e elaboração de mapas krigados para representar a altitude obtida pelos dados GNSS, ASTER e SRTM (Figs. 4A, 4B e 4C).

Carvalho et al. (2012) também observaram melhor ajuste com o modelo esférico para a altitude do estado de São Paulo. Esses autores testaram dois conjuntos de dados, um com 1027 observações e outro com 445 observações, sendo que quanto maior o número de observações, maior foi o alcance. Fenille e Cardim (2007) analisaram a variabilidade espacial da altitude e das precipitações pluviométricas para o estado de São Paulo e observaram ajuste com o modelo exponencial para a altitude.
Por meio do semivariograma esférico, observou-se que o alcance foi crescente para a altitude, com alcance de 317,60 para GNSS, de 979,69 para ASTER e de 998,74 m para SRTM (Tab. 2), sendo importante ressaltar que quanto menor a distância de um ponto para o outro com diferente valor de altitude, menor a dependência final dos dados de altitude.

Foram observados por meio da krigagem, os maiores valores de altitude na região leste do campus da UFMT e os menores valores na região noroeste, principalmente na marginal ao curso do córrego do Barbado, para todas as bases de dados testadas (Figs. 4A, 4B e 4C). 

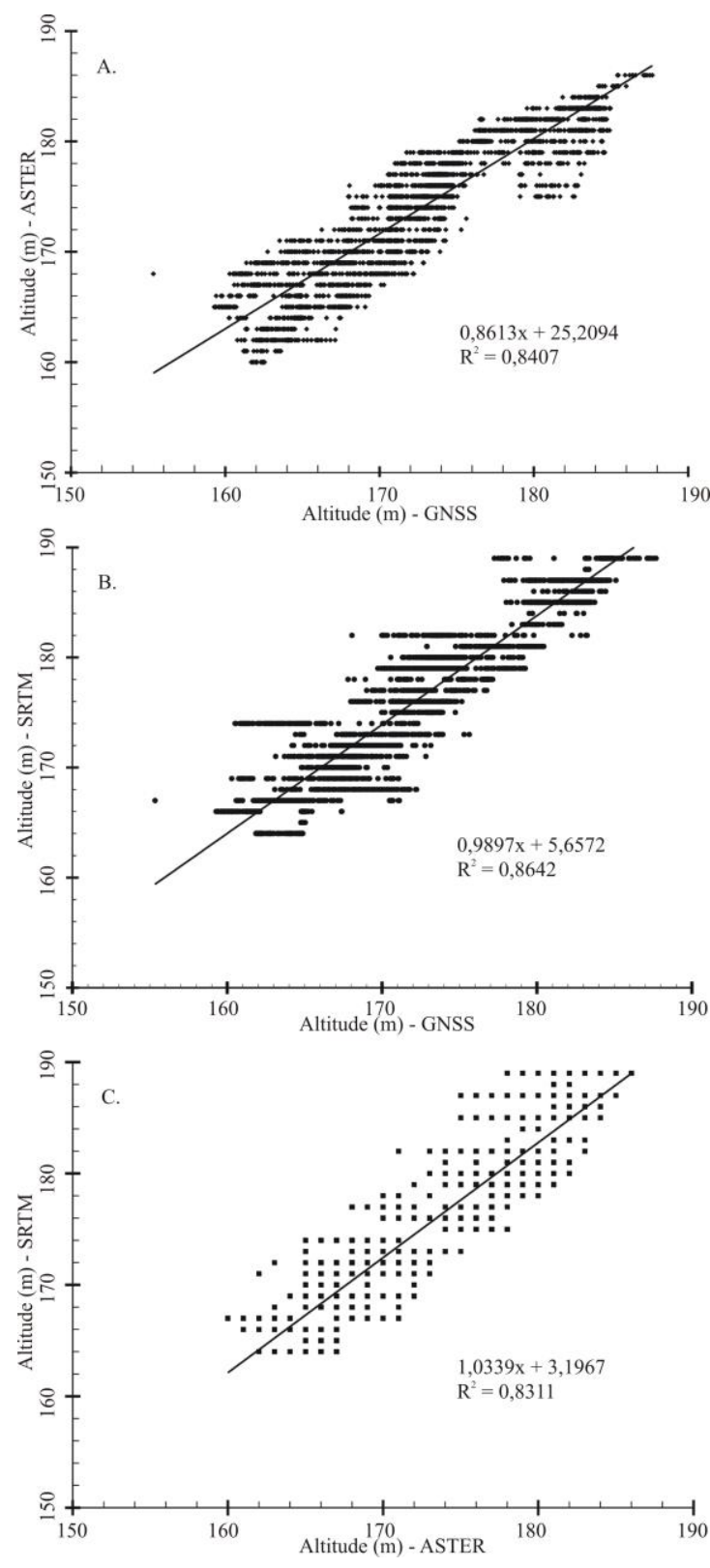

Figura 2 - Relação entre os valores da altitude obtidos por meio de GNSS e o ASTER GDEM (A), GNSS e o SRTM (B) e ASTER GDEM e o SRTM (C).
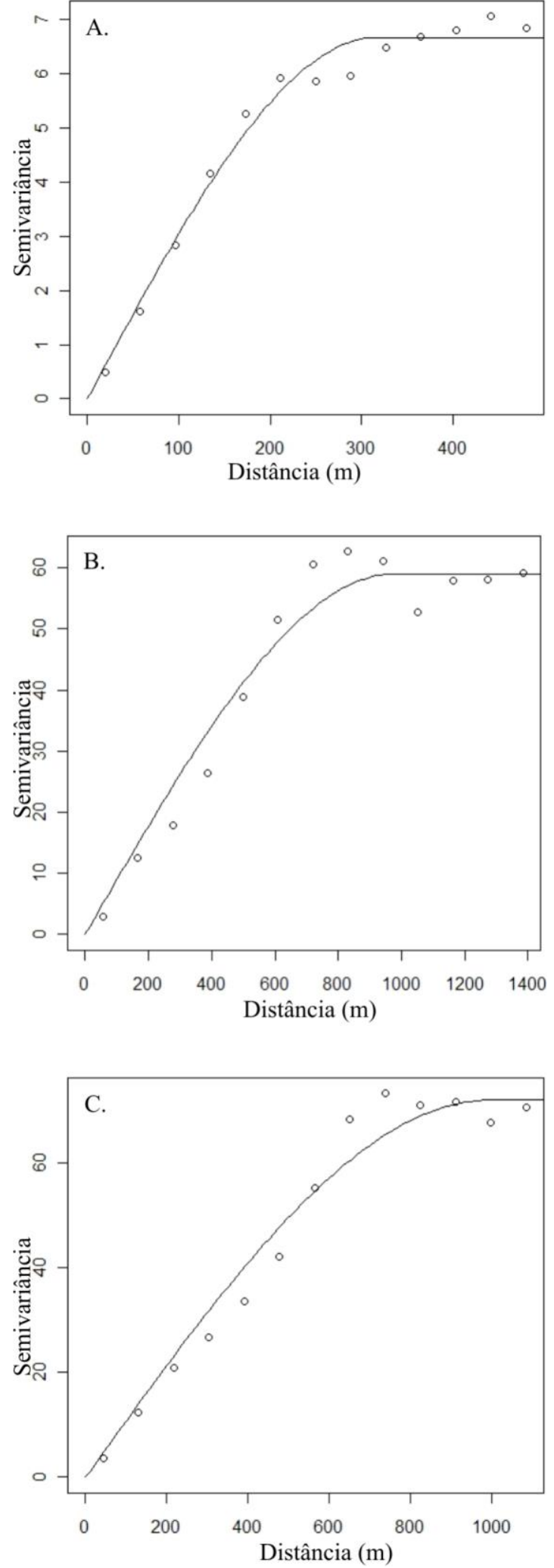

Figura 3 - Semivariogrma esférico da altitude referente aos dados do GNSS (A), ASTER GDEM (B) e SRTM (C).

A ilustração do mapa de krigagem da altitude contribuirá com informações para o planejamento territorial do campus da UFMT.

Esses resultados estão de acordo com aqueles obtidos por Grohmann e Steiner (2008), que testaram 
splines, ponderação pelo inverso da distância e krigagem para o MDE na região sudeste da Califórnia e observaram que a qualidade do resultado final foi considerada satisfatória com a utilização da krigagem. Valeriano et al. (2006), ao estudarem a morfometria de pequenas bacias de drenagem na Amazônia, testaram métodos de interpolação e também observaram melhores resultados com a utilização da krigagem.

Os maiores erros da krigagem foram observados onde houve menor amostragem de pontos (Figs. 5A, 5B e 5C). Resultados semelhantes foram obtidos por Montebeller et al. (2007) em estudo sobre a variabilidade espacial da erosividade no estado do Rio de Janeiro, tendo observado que as áreas com maiores variâncias predominaram nas regiões litorâneas e norte, as quais apresentaram maior distância entre os pontos amostrais.

A menor amplitude do erro ocorreu com os dados GNSS e a maior amplitude do erro foi verificada com os dados do SRTM (Figs. 5A, 5B e 5C), que apresentou maior erro especialmente na região sudoeste, onde há um bosque com várias árvores, e na região norte e noroeste, sendo que nessas áreas há uma extensa área com construção civil e uma represa. É importante destacar que esse erro não ultrapassou 2,5 m (Fig. 5C), Rodriguez et al. (2006) afirmaram que, para a América do Sul, os dados do SRTM têm precisão vertical de $6,2 \mathrm{~m}$ e horizontal de 9,0 m.

Entre os fatores que podem influenciar nos dados SRTM, quando analisada a altitude, Koch e Lohmann (2000) destacaram a influência da vegetação e da cobertura do solo. Os dados SRTM são utilizados na maioria das pesquisas que envolvem altitude, embora nem todos os resultados apresentados sejam superiores aos demais conjuntos de dados testados. Em estudo conduzido por Carvalho et al. (2004), os resultados do relevo obtidos por SRTM foram satisfatórios em áreas com relevo acidentado, porém geraram problemas na determinação de declividades em áreas planas. Chagas et al. (2010) avaliaram MDEs e observaram que o MDE SRTM apresentou resultados bastante satisfatórios, mas ligeiramente inferiores aos MDE obtido por meio de dados de curva de nível e ASTER.

Estudos realizados por Tomazoni et al. (2011), também observaram que a vegetação das matas ciliares são captadas pelo SRTM e ASTER e interferem nas variações de altitude e por conseguinte no mau delineamento das redes de drenagem. Czubsi et al. (2013) estudaram a acurácia dos dados STRM e ASTER, e verificaram que embora ambos os modelos apresentarem descrição estatística confiável da superfície do terreno, eles exigem correções adicionais para modelagem da hidrologia ou do terreno.

A geoestatística possibilitou caracterizar a estrutura e a magnitude de dependência espacial das três bases cartográficas e interpolar os dados com base nessa estrutura (Figs. 3 e 4). Com isso, foi possível avaliar os erros de cada modelo na área do campus, suavizando eventuais discrepâncias de estruturas prediais e árvores não eliminadas pelos modelos ASTER e SRTM. Outros erros referentes aos mecanismos e processos de obtenção de dados foram determinados pelo mapa de erro da krigagem e suavizados no mapa de krigagem (Fig. 5).
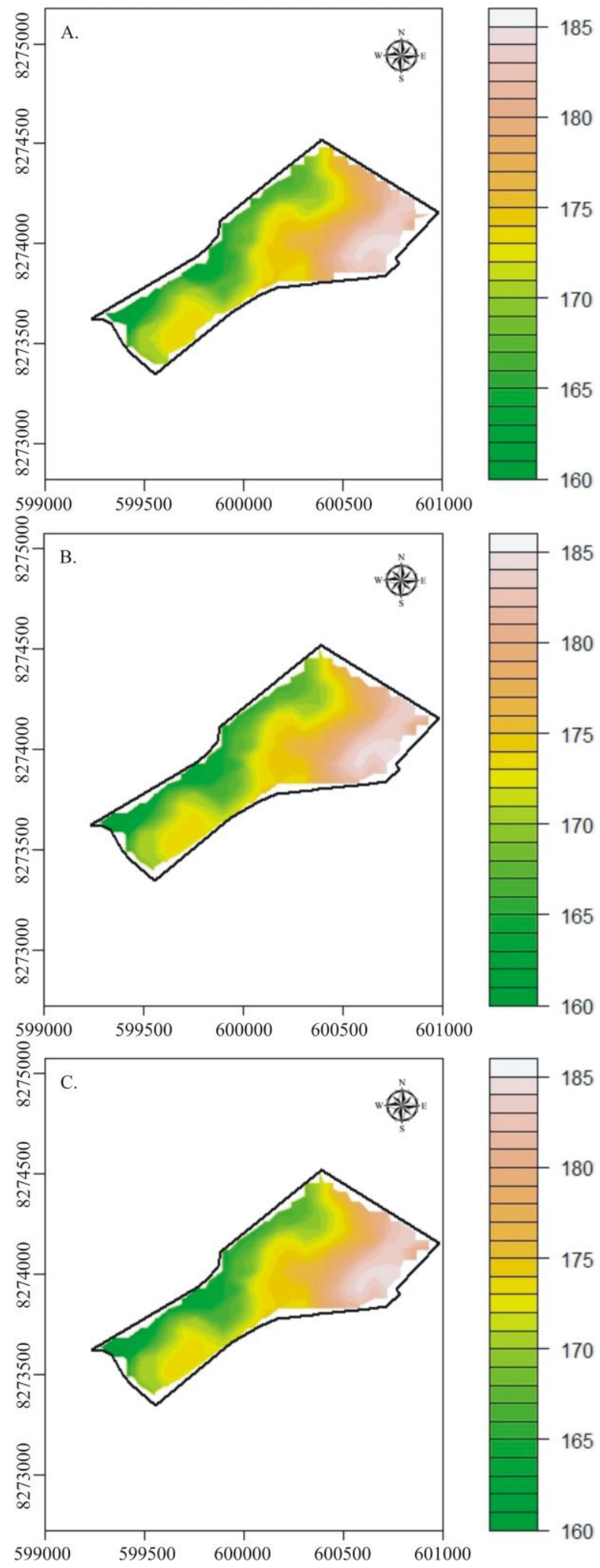

Figura 4 - Krigagem ordinária da altitude referente aos dados do GNSS (A), ASTER GDEM (B) e SRTM (C). 

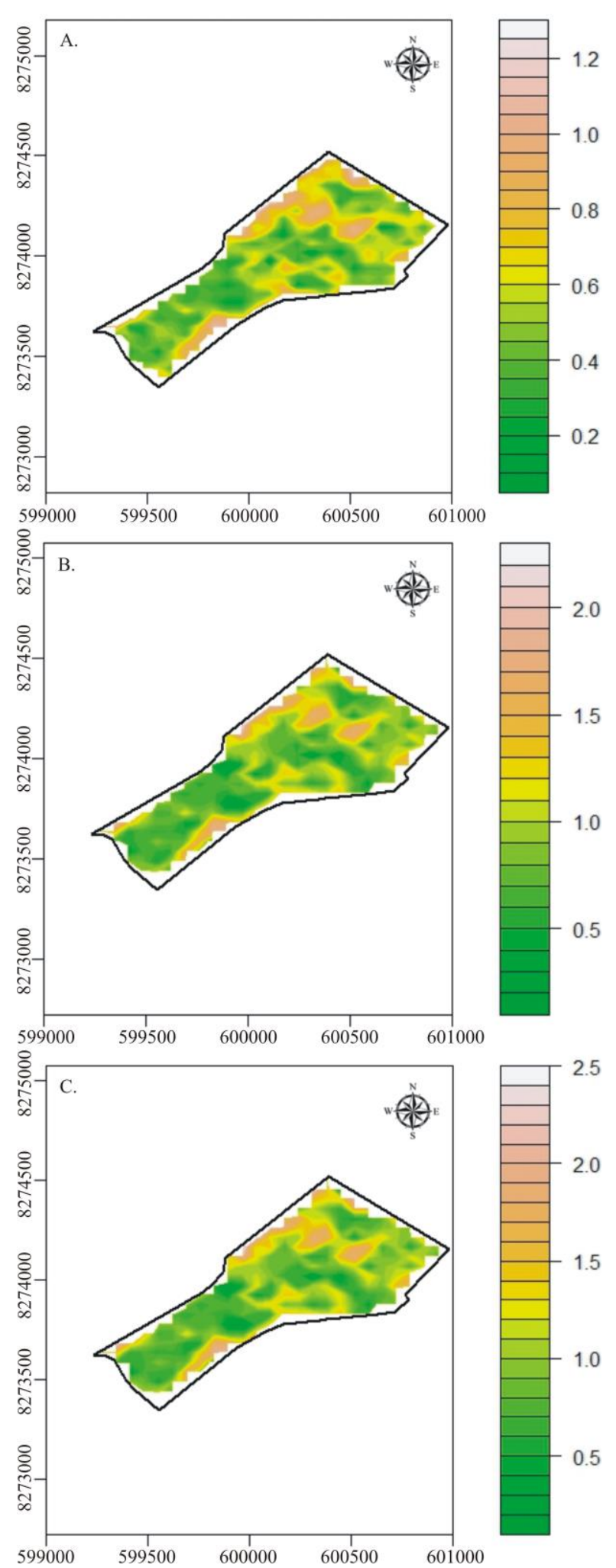

Figura 5 - Erro da krigagem da altitude referente aos dados do GNSS (A), ASTER GDEM (B) e SRTM (C).

Praticamente os mapas krigados não apresentaram visualmente diferença, mas, pela observação de cada conjunto de dados juntamente com a krigagem e, com o mapa de erro, foi possível verificar que os dados oriundos do GNSS representaram com mais detalhes a altitude da área estudada. Isso ocorreu possivelmente pela menor discrepância dos dados, os quais foram obtidos apenas na superfície e, portanto, com características de estacionariedade mais aplicáveis na interpolação de dados do que no caso dos dados derivados dos dados remotos.

\section{CONCLUSÃO}

Por meio da geoestatística, foi possível representar a variabilidade espacial da altitude do campus da Universidade Federal de Mato Grosso.

Para os dados do GNSS, ASTER GDEM e SRTM, o melhor modelo de semivariograma foi o esférico.

Os maiores erros da krigagem foram obtidos pelos dados SRTM e, os menores erros pelos dados GNSS.

\section{REFERÊNCIAS BIBLIOGRÁFICAS}

AREFI, H.; REINARTZ, P. Accuracy enhancement of Aster global digital elevation models using ICESat data. Remote Sensing, v.3, p.1323-1343, 2011.

BARBOSA, A. P.; SILVA, A. F. DA; ZIMBACK, C. R. L. Modelo numérico do terreno obtido por diferentes métodos em cartas planialtimétricas. Revista Brasileira de Engenharia Agrícola e Ambiental, v.16, p.655-660, 2012.

BURROUGH, P.A.; MCDONNELL, R.A. Principles of geographical information systems. 2nd Edition. New York: Oxford University Press, 1998. 333p.

CAMARGO, F. F.; ALMEIDA, C. M.; FLORENZANO, T. G.; OLIVEIRA, C. G. Acurácia posicional de MDEs ASTER/Terra em área montanhosa. Revista Geomática. v.4, p.12-24. 2009.

CARVALHO, J. R. P. de; ASSAD, E. D.; PINTO, H. S. Interpoladores geoestatísticos na análise da distribuição espacial da precipitação anual e de sua relação com altitude. Pesquisa Agropecuária Brasileira, v.47, p.1235-1242, 2012.

CARVAlHO, T. M. de; LATRUBESSE, E. M. Aplicação de modelos digitais do terreno (MDT) em análises macrogeomorfológicas: o caso da bacia hidrográfica do Araguaia. Revista Brasileira de Geomorfologia, ano 5, p.85-93, 2004.

CAMBARDELLA, C. A.; MOORMAN, T. B.; NOVAK, J. M.; PARKIN, T. B.; KARLEN, D. L.; TURCO, R. F.; KONOPKA, A. E. Field-scale variability of soil properties in Central Iowa soils. Soil Science Society of America Journal, v.58, p.1501-1511, 1994.

CHAGAS, C. S.; FERNANDES FILHO E. I.; ROCHA, M. F.; CARVALHO JÚNIOR, W.; SOUZA NETO, N. C. Avaliação de modelos digitais de elevação para aplicação em um mapeamento digital de solos. Revista Brasileira de Engenharia Agrícola e Ambiental, v.14, p.218-226, 2010.

CZUBSKI, K.; KOZAK, J.; KOLECKA, N. Accuracy of SRTM-X and ASTER elevation data and its influence topographical and hydrological modeling: case study of the Pieniny Mts. in Poland. International Journal of Geoinformatics, v.9, p.7-14, 2013.

DIGGLE, P. J.; RIBEIRO JUNIOR, P. J. Model-based Geoestatistics. New York: Springer, 2007. 228p. 
FALORNI, G.; TELES, V.; VIVONI, E. R.; BRAS, R. L.; AMARATUNGA, K. Analysis and characterization of the vertical accuracy of digital elevation models from the shuttle radar topography mission. Journal of Geophysical Research, v.110, p.1-20, 2005.

FELICÍSIMO, A. M. Modelos digitales del terreno: Introducción aplicaciones en las ciencias ambientales. Oviedo: Universidad de Oviedo, 1994. Disponível em: <http://www6.uniovi.es/ feli/pdf/libromdt.pdf>. Acesso em: 22 mai. 2013.

FENILLE, M. C.; CARDIM, M. Correlação espacial entre a altitude e as precipitações pluviométricas no estado de São Paulo. Geociências, v.26, p.35-141, 2007.

GHILANI, C. D.; WOLF, P.R. Elementary Surveying: An introduction to geomatics, 13th Edition. Pearson Education, Upper Saddle, NJ, 2012. 933p.

GROHMANN, C. H.; STEINER, S.S. SRTM resample with short distance-low nugget kriging. International Journal of Geographical Information Science, v.22, p.895-906, 2008.

HENGL, T.; BAJAT, B.; BLAGOJEVIC, D.; REUTER, H. I. Geostatistical modeling of topography using auxiliary maps. Computers \& Geosciences, v.34, p.1886-1899, 2008.

KERVYN, M.; ERNST, G. G. J.; GOOSSENS, R.; JACOBS, P. Mapping volcano topography with remote sensing: ASTER vs. SRTM. International Journal of Remote Sensing, v.29, p.6515-6538, 2008.

$\mathrm{KOCH}$, A.; LOHMANN, P. Quality assessment and validation of digital surface models derived from the shuttle radar topography mission (SRTM). IAPRS, v. XXXIII, Amsterdam, 2000.

MELLO, J. M.; BATISTA, J. L. F.; RIBEIRO JÚNIOR, P. J.; OLIVEIRA, M. S. de. Ajuste e seleção de modelos espaciais de semivariograma visando à estimativa volumétrica de Eucalyptus grandis. Scientia Forestalis, v.1, p.25-37, 2005.

MONTEBELLER, C. A.; CEDDIA, M. B.; CARVALHO, D. F.; VIEIRA, S. R.; FRANCO, E. M. Variabilidade espacial do potencial erosivo das chuvas no estado do Rio de Janeiro. Engenharia Agrícola, v.27, p.426-435, 2007.

NIKOLAKOPOULOS, K. G.; KAMARATAKIS, E. K.; CHRYSOULLAKIS, N. SRTM vs ASTER elevation products. Comparison for two regions in Crete, Greece. International Journal of Remote Sensing, v.27, p.4819-4838, 2006.

TOMAZONI, J. C.; GUIMARÃES E.; GOMES, T. C.; SILVA, T. G da. Uso de modelo digital de elevação gerados a partir do ASTER GDEM e SRTM para caracterização de rede de drenagem. Revista Brasileira de Geografia Física. n.2. p.365-376. 2011.

RODRIGUEZ, E.; MORRIS, C. H.; BELZ, J. E. A global assessment of the SRTM performance. Photogrammetric Engineering \& Remote Sensing, v.72, p.249-260, 2006.

SMITH, B.; SANDWELL, D. Accuracy and resolution of shuttle radar topography mission data. Geophysical Research Letters, v.30, p.1-4, 2003.
STEVENS, N. F.; GARBEIL, H.; MOUGINIS-MARK, P. J. NASA EOS Terra ASTER: Volcanic topographic mapping and capability. Remote Sensing of Environment, v.90, p.405-414, 2004.

VALERIANO, M. M.; KUPLICH, T. M.; STORINO; M. AMARAL, B. D.; MENDES, J. N.; LIMA D. J. Modeling small watersheds in Brazilian Amazonia with shuttle radar topographic mission-90 m data. Computers and Geoscience, v.32, p.1169-1181, 2006.

WARRICK, A.W.; NIELSEN, D. R. Spatial variability of soil physical properties in the field. In: Hillel, D. Applications of soil physics. New York: Academic Press, 1980. p.319-344. 